\title{
Analytical hierarchy process based flexibility measurement tool to integrate concentrated photovoltaic in smart grid
}

\author{
Ahmed A. Shetaya ${ }^{\mathrm{a}, \mathrm{b} *}$, Rasha El-Azab ${ }^{\mathrm{a}, \mathrm{b}}$, Amr M. Amin ${ }^{\mathrm{a}, \mathrm{b}}$ \\ ${ }^{a}$ Department of Electrical Power Engineering, Faculty of Engineering, Helwan University, Cairo, 11792, Egypt \\ ${ }^{b}$ Academy of Scientific Research and Technology, Al-Kasr El-Aynay, Cairo, 11516, Egypt
}

\begin{abstract}
Sustainable Energy Sources have many challenges that significantly depend on the specific features of each renewable variable type as wind, Photovoltaic (PV), Concentrated Solar Power (CSP) and Concentrated Photovoltaic $(\mathrm{CPV})$. These new generation technologies have many complications that linked with power system integration as variability and uncertainty environment. This paper introduces a new process based on Analytical Hierarchy Process (AHP) to aid the integrating of CPV in Smart Grids. New smart power systems behavior is affected by the new composite CPV generation and loads that have uncertain and variable nature. The recommended technique measures current grid flexibility index, and checks its capability to operate grid securely based on Smart Grid wide area measurements. It also determines the desired storage that must be added to enhance the system flexibility for real time dispatching.
\end{abstract}

Keywords: Analytic Hierarchy Process (AHP), Concentrated Photovoltaic (CPV), Energy Storage Systems (ESS), power system flexibility, smart grid.

\section{Introduction}

Nowadays, the conventional fossil fuel will be significantly consumed. All over the world, new alternatives Renewable Energy Sources (RES) will be used to cover the big shortage in traditional energy sources. The different energy sources types ensure reliability of the power system, but it will cause uncertainty and variability of the power sources issues.

The key principle of CPV usage over traditional PV or CSP is the cost efficient concentrating optics that decreases the area of high costly, high efficiency [1]. While in many areas in the Middle East as an example have sun-rich with direct normal irradiance. So, the usage of CPV is more efficient than another solar power system [2]. Power system flexibility is defined as the capability of a grid to dispatch its resources to accommodate the changes in net load, where net load is defined as the residual of grid load that not served by variable generation.

The variety of generation sources have different flexibility limit in the smart grid. Therefore, the need to measure the flexibility impact of CPV becomes very essential. An accurate index is needed to evaluate the CPV impact and define the required traditional reserve and/or storage system [2].

There are many Frameworks and metrics for measuring power system flexibility are evolving. In [3], flexibility charts, are developed to deliver a snapshot overview of different types of generation-based flexibility at each country. These charts work offline to give useful information only for planners. They do not share in real time operation of the system.

Juan Ma, [4] introduces sampled flexibility measurement to evaluate and plan flexibility. But, the proposed technique is depends only on ramp rate, minimum and maximum power. Startup time and

\footnotetext{
* Manuscript received April 27, 2015; revised August 15, 2015.

Corresponding author. Tel.: +20-122-263-7779; E-mail address: ahmed.shetaya@ @-eng.helwan.edu.eg.

doi: $10.12720 /$ sgce.4.3.233-240
} 
controllability criteria great impacts are neglected on the flexibility evaluation. AHP is a structured technique for organizing and analyzing complex decisions, based on mathematics. There are many application of AHP as in ranking, choosing, prioritization, resources allocation and management.

In this paper, the proposed technique is based on the Analytic Hierarchy Process (AHP). Consequently, the type and size of the flexibility sources (Conventional/Energy Storage Systems) will be defined, via an optimal efficient way, to increase the profit. The proposed metric tool could be used to measure impact of CPV sharing in the smart grid on operation flexibility and introduce guidelines for planners to be aware when adding ESS.

\section{CPV Dispatching Difficulties}

CPV generation promises higher efficiency compared to flat traditional PV systems or CSP system, about $43 \%$. CPV has low capital cost. As, CPV consumes less semiconductor raw materials compared to $\mathrm{PV}$. In addition to, the area that needed to install CPV is smaller than area that needed for PV one. Also, high energy capacity factor (KWh/ installed KW) that make the CPV beat PV or CSP [1].

The yearly net load ramp rate (MW/hour) has high ramp rate during most of the year due to high CPV penetration. Dispatching of demand power is hard to be predicted or controlled. The expected high ramp rate in demand power needs high flexible sources or reserves to cover the shortage of the CPV output. Due to the variability of the CPV output, the dispatcher is forced to curtail the CPV power in many cases to operate the power system safely. CPV curtailment causes losing a free power, and reduces the system profit [5]-[7]. To overcome all the challenges of the CPV penetration, the conventional reserve that driven from traditional sources as Steam, Gas or Hydro power generation should be used. By adequate dispatching between these traditional sources, any power shortages during CPV operation can be covered [8].

The conventional reserve sources have different flexibility characteristics and natures. Therefore, a flexibility index must be measured, to evaluate their appropriateness in covering the CPV power shortage. According to the flexibility measurement, the operator may use Energy Storage System, as extra source for the reserve, to follow the power demand ramping and enhance flexibility [9].

\section{Energy Storage Systems}

Energy storage systems (ESS) provide more flexibility for the smart grid operation. There are many storage technologies used in power system such as, Pumped Hydro Storage (PHS), Compressed Air Energy Storage (CAES), Thermal Energy Storage (TES), Batteries, flywheels and super-capacitors technology.

The specifications of ESS are different with respect to unlike aspects. There are storage type used to follow the power changes in CPV uncertain and variable power as PHS, CAES, TES and Batteries. Other types are used to smooth power fluctuation in small duration, and improve power quality of the power systems as Batteries, flywheels or super-capacitors [10]. On the other hand, the maturity of the storage technologies varies from type to another. In [11], PHS, TES and CAES are considered as more economical and commercial in following power variety than others. While, batteries, flywheels or supercapacitor are in demonstration stage. PHS is available with low cost with high power sharing. While, thermal storage system has ability to shift the power of CPV or CSP generation to peak-hour time.

In last five years, the battery storage systems predicted to be greatly growth. Also, the new researches improve battery usage in medium and high power scales. So, it would be used in follow power variability in CPV generation or in renewable sources [10]. In this study, battery storage systems, PHS and TES are studied, as a complete solution of CPV generation challenges. The results verify the capability of these storage systems of healing the system flexibility.

\section{Flexibility Evaluation Criteria}

The flexibility evaluation process depends on different criteria. From technical point of view, there are 
four criteria with large impacts on the flexibility. The minimum power of the generation unit, the ramp rate capability, cycling rate or startup time and controllability nature of the generating unit are the main characteristics of the flexibility. The following section discusses the importance of each criteria.

\subsection{Minimum power}

The minimum permissible power could reserve unit operate. It plays important role in measuring flexibility. If the minimum power of the generating or storage unit is large, it can't meet different power changes, due to large variability of the renewable energy sources with high sharing in the power system.

\subsection{Ramp rate}

The most important factor is the positive or negative ramp rate capability of the reserve source. It defines the degree of meet the ramp rate of the load minus renewable sources. The ramp rate is measured by $\mathrm{MW} / \mathrm{min}$. There are big verities in flexibility sources, from one can follow power in different time frame to another one that can regulate the power in very small time scale. Each unit must be utilized according to the dispatching process by depending on the flexibility measurement value and rate of change.

\subsection{Startup time}

The startup time gives information about how fast the power unit commitment. It plays a strong role during large change in the load or large variety in the uncertain variable generation sources.

\subsection{Controllability}

The criteria of controllability depend on different characteristics and factors that may limit the usage of generating or storage unit. For example, the usage of Nuclear power plant is difficult during power dispatching and operation due to safety and technical factor although the minimum power is low [12]-[15].

Fig. 1 shows proposed analytical hierarchy diagram in designing the flexibility metric tool. Sources of flexibility are listed in the last layer. Last layer defines all generation /storage alternates. Each type of alternates has its own criteria or specifications in the middle criteria layer. Each criteria source has specific weights that share in the proposed flexibility index.

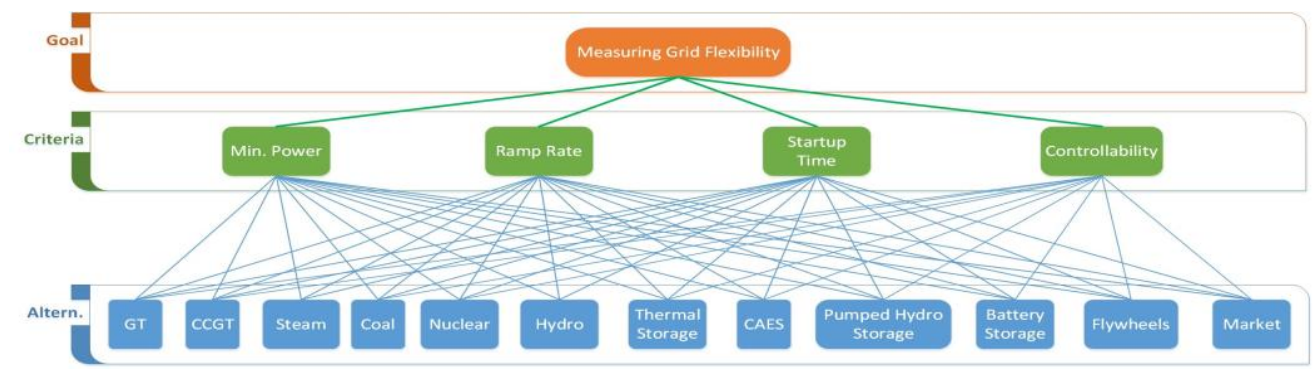

Fig. 1. Flexibility evaluation criteria based on AHP.

Proposed flexibility tool ranks all alternatives from the best the worst flexible scenario for real time operation. The following section discusses how the proposed algorithm of AHP is used to solve multicriteria problem to measure the flexibility index.

Also it is ranked from the best flexibility to the worst to be used during real time operation. The following section shows how the proposed algorithm of AHP is used to solve multi-criteria problem to measure flexibility index.

\section{Analytical Hierarchy Process}

The measurement of flexibility is built according to the four criteria that defined previously. Each 
criteria has its own priority in case flexibility measurement. To make decision of organizing the criteria according to their impact on flexibility, the choice should be decomposed into the following steps [16].

- Define the problem and find the available known specification and build the structure of the hierarchy from the goal through the main four criteria to lower level of alternates.

- Construct a set pairwise comparison matrices with respect two each criteria and each criteria with respect to the main goal.

- Use the priorities to weight each alternate and to weight each criteria.

- Rank the alternates according to the goal to evaluate upon the available alternates and their weights. Suppose that $n$, alternates, $A_{1}, \ldots . ., A_{n}$, whose weights $\omega_{1}, \ldots . ., \omega_{n}$, respectively, are known. The pairwise matrix contains the ratio of weights according to the fundamental scale. The comparison is made between each two alternates and the inverse of the comparison is made according to reciprocal of the weights as in the following matrix equation:

$$
\left(\begin{array}{cccc}
\omega_{1} / \omega_{1} & \omega_{1} / \omega_{2} & \cdots & \omega_{1} / \omega_{n} \\
\omega_{2} / \omega_{1} & \omega_{2} / \omega_{2} & \cdots & \omega_{2} / \omega_{n} \\
\vdots & \vdots & \vdots & \vdots \\
\omega_{n} / \omega_{1} & \omega_{n} / \omega_{2} & \cdots & \omega_{n} / \omega_{n}
\end{array}\right)\left(\begin{array}{c}
\omega_{1} \\
\omega_{2} \\
\vdots \\
\omega_{n}
\end{array}\right)=n\left(\begin{array}{c}
\omega_{1} \\
\omega_{2} \\
\vdots \\
\omega_{n}
\end{array}\right)
$$

It could be summarized as:

$$
A \omega=n \omega
$$

The pairwise comparison matrices in AHP is based on the fundamental scale of intensity of importance, as shown in Fig. 2 [16].

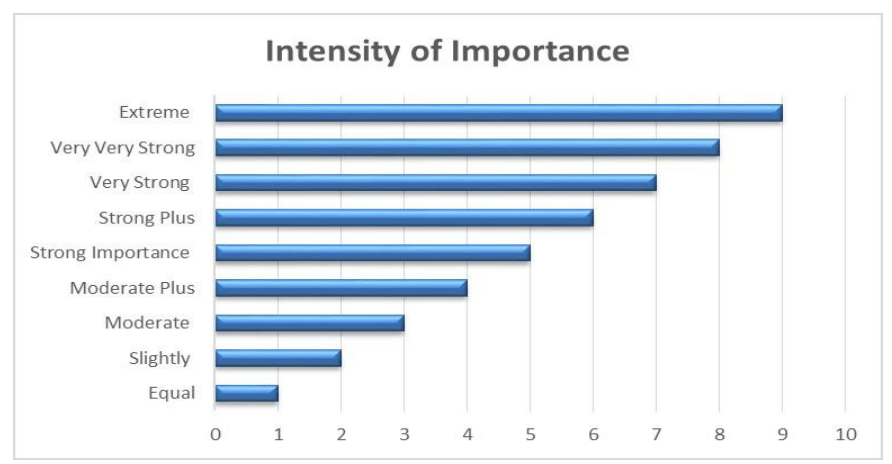

Fig. 2. AHP fundamental scale and random consistency index.

The solution of (2) is called the principle right eigenvector of $A$, . To make weights $\omega$, unique; the entries must be normalized by dividing over their sum. After priorities calculation, the opinion must be measured to verify it's consistent. The consistency may be measured for judgment of solution by the following consistency index in (3).

$$
C I=\frac{\lambda_{\max }-n}{n-1}
$$

where, $\lambda_{\max }$ is the largest principle eigenvalue in the eigenvector. The consistency index (CI) must be compared to random consistency index (RI) that is pre-calculated in [12]. Then, the consistency ratio is 
calculated as in (4).

$$
C R=\frac{C I}{R I}
$$

If the value of the consistency ratio is smaller than or equal to $10 \%$, the inconsistency is accepted. While the ratio in (4) will be used for each alternates with respect to criteria and also in case of comparing the criteria with respect to the goal.

\section{AHP Implementation to Evaluate CPV Inflexibility Impact}

The AHP is proposed to measure the flexibility of each reserve source and evaluate the inflexibility level of the CPV to estimate the grid needs of flexibility after sharing of CPV. The available reserve flexibility depends on the pre-discussed main criteria. The full specifications of each generation or storage type are listed in Table 1, [12]-[15].

The minimum power criteria define the minimum permissible power that taken from each reserve. The second criterion is the ramp rate capability of generation/ storage units that considered as the most significant factor directly affect on the flexibility. The ability of the reserve source to follow the ramp rate of CPV generation is vital factor to measure the flexibility. In Gas Turbine (GT) and in Combined Cycle Gas Turbine (CCGT), the ramp rate lies between 10 to $20 \%$ of size/ Minutes. But in the steam turbine and coal based power plant, is less ramp rate.

On the other hand, the nuclear power plant is difficult to follow the ramp rate of the net load because the ramp rate is approximately $20 \%$ of size/Minutes.

Table 1: The reserve sources specification with respect to main criteria

\begin{tabular}{|c|c|c|c|c|}
\hline Reserve Sources & $\begin{array}{c}\text { Minimum } \\
\text { Power } \\
\text { (\% of Size) }\end{array}$ & $\begin{array}{c}\text { Ramp Rate } \\
\text { (\% of } \\
\text { Size/Min) }\end{array}$ & $\begin{array}{c}\text { Start Up } \\
\text { Time } \\
\text { (Min.) }\end{array}$ & Controllability \\
\hline GT & 40 & 20 & 15 & Close to Full \\
\hline CCGT & 40 & 10 & 45 & Half \\
\hline Steam Turbine & 40 & 5 & 300 & Low \\
\hline Coal & 50 & 5 & 300 & Half \\
\hline Nuclear & 50 & 2 & 2400 & Very Low \\
\hline Hydro & 15 & 30 & 15 & Close to Full \\
\hline Pumped Hydro Storage & 15 & 30 & 15 & Close to Full \\
\hline Battery Storage & 0 & 100 & 0 & Full \\
\hline Thermal Storage & 75 & 20 & 15 & High \\
\hline CAES & 0 & 100 & 2 & Full \\
\hline Flywheels & 0 & 100 & 0 & Full \\
\hline Market & 0 & 100 & 0 & Full \\
\hline
\end{tabular}

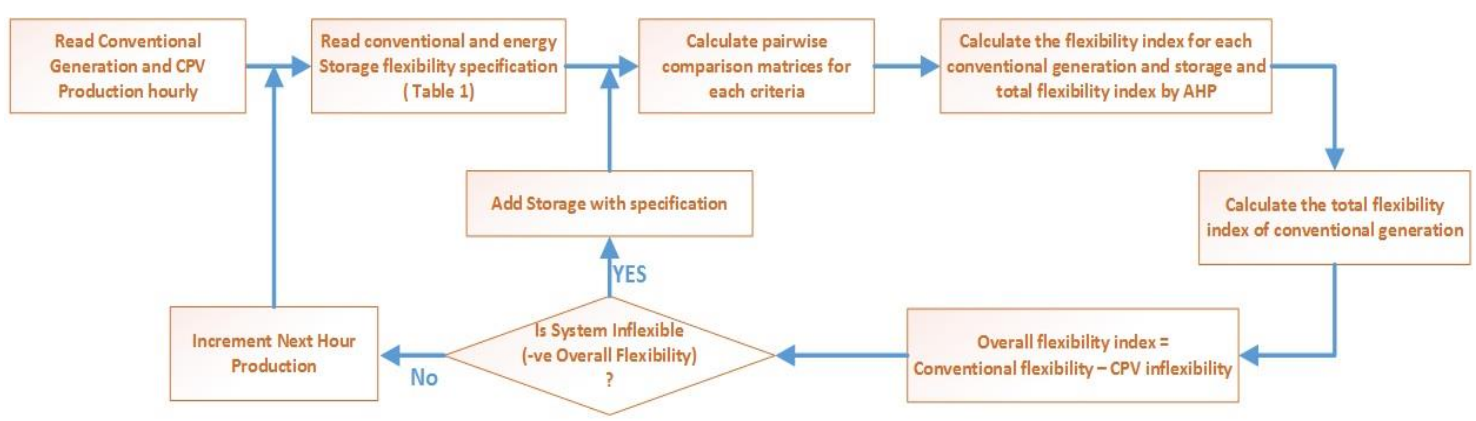

Fig. 3. Flow diagram of the flexibility metric based on AHP.

In Hydro power plant, the ramp rate is greater which reach to about 30\%/Minutes, and in the other storage system is full dispachable. The third criteria is the startup time that shown in table 1 [12]-[15]. 
Another significant factor of the flexibility measurement goal is the controllability of the resources. GT, hydro plant and pumped hydro plant are close to be full dispachable, while, CCGT and Coal are half controlled due to its dependence on the steam boiler operation. In the thermal storage, the system is high enough to be dispatched in some limitation. But in the nuclear power plant, it is very difficult to be controlled while in the other side, the storage system and the market is nearly full controllable. Fig. 3 shows the flow diagram of the calculation steps of overall flexibility of the power system after adding the impact of CPV generation.

First, the algorithm reads the hourly production of the conventional generation and CPV generation. Then, the proposed technique delivers the specifications of the conventional generation units and storage systems that in Table 1. After that, the flexibility index of the each conventional generation, storage and total reserve is calculated by using AHP. The source of inflexibility in this case is the CPV generation. AHP will calculate the inflexibility index of it based on its sharing value to the total generated power. At that point, the overall flexibility is calculated by subtracting the flexibility index of conventional sources and storage from the inflexibility index of CPV generation. Finally, the algorithm will check if the system is inflexible (negative overall flexibility), the system propose to add extra storage unit or size from the available type of the system according to its flexibility ranking and from economical point of view.

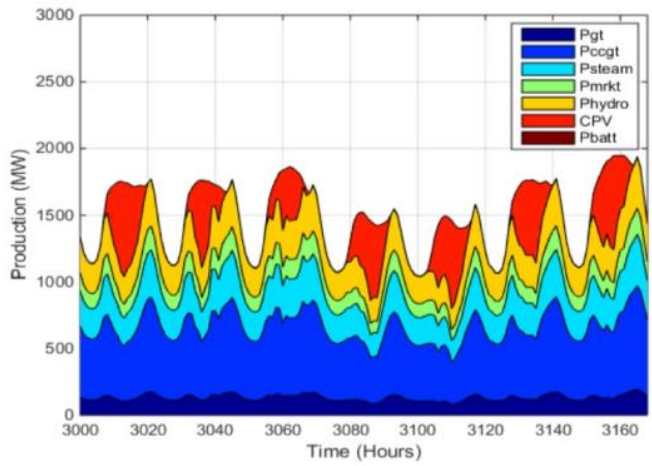

(a)

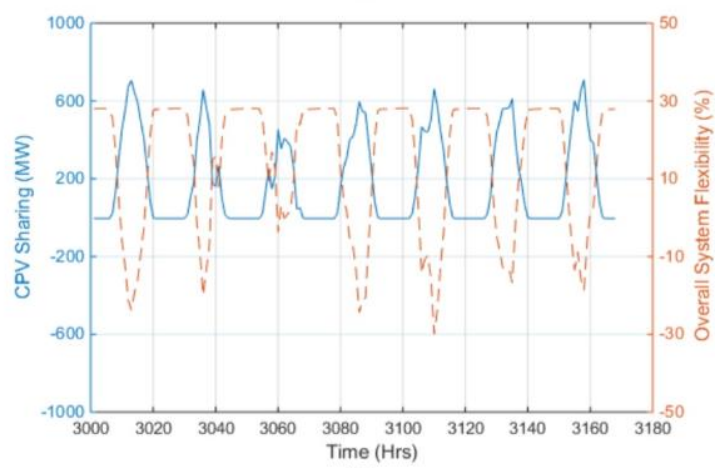

(b)

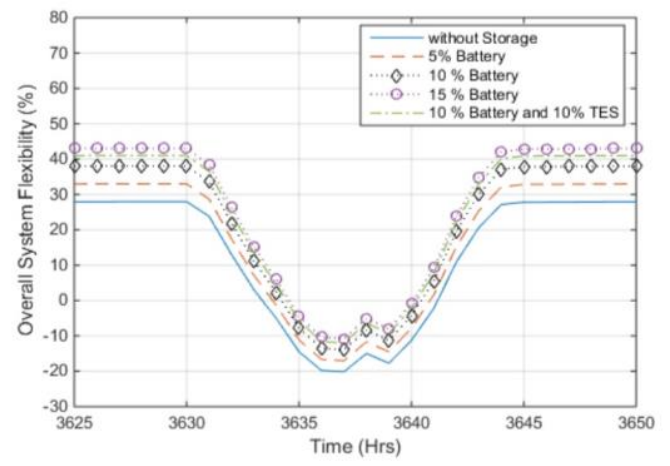

(c)

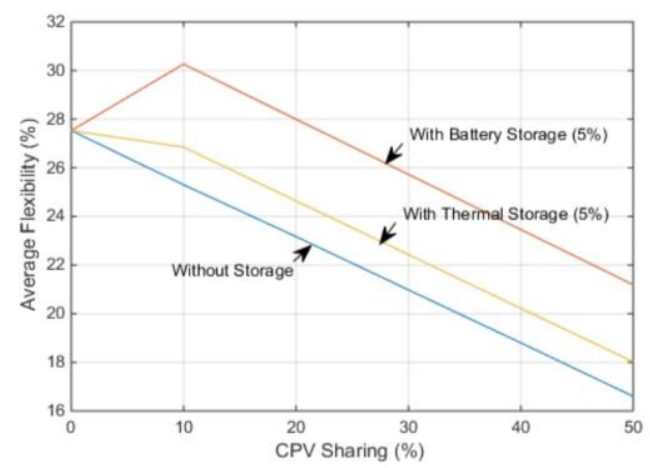

(d)

Fig. 4. CPV impact case study: (a) production (MW), (b) overall flexibility vs. CPV sharing, (c) $40 \%$ CPV at worst day with different storage and (d) average flexibility at different CPV sharing.

\section{Case Study and Results Discussion}

The hourly production of different generation types for 5 days (120 hours) are given as an example to estimate the sources overall flexibility. Fig. 4(a) shows the sharing of sources as GT, CCGT, steam power plant, hydro generation and market sharing. In addition two sharing of CPV generation by about $50 \%$ of total sum of the load. After the algorithm is applied on the system, the overall flexibility index verses CPV sharing is shown in Fig. 4(b). 
Fig. 4(c) shows the worst day in the year. The flexibility is evaluated without adding any storage to the system. By adding $5 \%$ battery storage to the system, the flexibility performance is improve by about $4 \%$, as shown in Fig. 4(c). On the other hand, by adding a mix of $10 \%$ battery and $10 \%$ thermal storage to the system, the flexibility is improved less than the case of adding $15 \%$ battery.

Fig. 4(d) shows the evaluation of CPV impact with different sharing by steps from $10 \%$ to $50 \% \mathrm{CPV}$ penetration. The figure shows the base case without storage and another two cases of adding $5 \%$ battery storage and 5\% TES.

Another solution for the flexibility is to curtail a part of CPV during a part of a day to reduce the cost of the storage system. The feasible study that based on the optimization of cost and technical behavior is made to optimize the operation of the grid with the high penetration of the CPV. So, the proposed flexibility metric tool must be taken into account of the optimal dispatching of the power as a very effective tool to retrieve the technical properties of the generation and storage systems.

\section{Conclusion}

CPV generation impact on power system flexibility has been evaluated. An AHP based technique is proposed to measure this impact. Proposed technique introduces the solution of covering expected flexibility shortage via selecting and evaluating different storage system. The results show that, the CPV has a great impact on the flexibility and the impact linearly increased by increasing CPV sharing. Also, the required storage system as battery and thermal storage system is evaluated to improve flexibility performance. Hybrid energy storage system with small CPV curtailment is also studied and evaluated. In the future research work, the factor of energy cost will be repossessed to evaluate the required storage impact with lower cost.

\section{Acknowledgements}

The authors gratefully acknowledge the grant and support of "Small scale thermal solar district units for Mediterranean communities", Priority 2 - Topic 2.3 Solar Energy, Ref. I-A/2.3/174, ENPI CBCMED. The research leading to these results has received funding from the European Union with Academy of Scientific Research and Technology.

\section{References}

[1] Kurtz SR. Opportunities and challenges for development of a mature concentrating photovoltaic power industry. National Renewable Energy Laboratory, 2009.

[2] Denholm P, Mehos M. Enabling greater penetration of solar power via the use of CSP with thermal energy storage. Solar Energy: Application, Economics, and Public Perception, 2014:99.

[3] Cochran J, Miller M, Zinaman O, Milligan M, Arent D, et al. Flexibility in 21st Century Power Systems. No. NREL/TP-6A2061721. National Renewable Energy Laboratory, Golden, CO., 2014.

[4] Ma J. Evaluating and planning flexibility in a sustainable power system with large wind penetration. PhD diss., The University of Manchester; 2012.

[5] Rady M, Amin AMA, Ahmed M. Development of small scale multi-generation concentrated solar plant for a medical center in Egypt. In: Proc. EUROSUNMED Symposium of Advanced Materials and Technologies for Renewable Energies, Lille, France, May 14-15, 2015.

[6] El Azab RM, Amin AMA. Optimal solar plant site selection. In: Proc. IEEE Southeast Con., Conference Proceedings, Fort Lauderdale, Florida USA, April 2015.

[7] Rahouma A, El-Azab R, Salib A, Amin AMA. Frequency response of a large-scale grid connected solar photovoltaic plant. In: Proc. IEEE Southeast Con., Conference Proceedings, Fort Lauderdale, Florida USA, April 2015.

[8] Amin AMA, et al. Concentrating solar power in Europe, the middle east and north Africa: achieving its potential. Journal of Energy and Power Engineering, February 2013; 7(2).

[9] Eto Joseph $\mathrm{H}$. Use of frequency response metrics to assess the planning and operating requirements for reliable integration of variable renewable generation. Lawrence Berkeley National Laboratory, 2011.

[10] IEA. Technology roadmap: energy storage technologies. [Online]. Available: https://cleanenergysolutions.org/training/ieaenergy-storage-technology-roadmap

[11] Khayyer P, Ozguner U. Decentralized control of large-scale storage-based renewable energy systems. IEEE Transactions on Smart Grid, May 2014; 5(3):1300-1307. 
[12] Load-Following Operating Mode at Nuclear Power Plants and Incidence on Operation and Maintenance (O\&M) Costs: Compatibility with Wind Power Variability. Publications Office; 2010.

[13] Frey GW, Linke DM. Hydropower as a renewable and sustainable energy resource meeting global energy challenges in a reasonable way. Energy Policy, 2002; 30(14):1261-1265.

[14] Eyer J, Garth C. Energy storage for the electricity grid: benefits and market potential assessment guide. Sandia National Laboratories, 2010:69-73.

[15] Peterseim JH, Hellwig U, Guthikonda M, Widera P. Quick start-up auxiliary boiler/heater-optimizing solar thermal performance. In: Proc. Solar PACES, 2012.

[16] Saaty TL. How to make a decision: the analytic hierarchy process. European Journal of Operational Research, 1990; 48(1):926. 